One-dimensional phased array with mechanical motion for conformal ultrasound hyperthermia

This article has been downloaded from IOPscience. Please scroll down to see the full text article.

2003 Phys. Med. Biol. 48167

(http://iopscience.iop.org/0031-9155/48/2/303)

The Table of Contents and more related content is available

Download details:

IP Address: 140.112.113.225

The article was downloaded on 06/01/2009 at 06:02

Please note that terms and conditions apply. 


\title{
One-dimensional phased array with mechanical motion for conformal ultrasound hyperthermia
}

\author{
Kuen-Cheng Ju${ }^{1}$, Yung-Yaw Chen ${ }^{1}$, Win-Li Lin ${ }^{2}$ and Te-Son Kuo ${ }^{1}$ \\ ${ }^{1}$ Department of Electrical Engineering, National Taiwan University, Taipei, Taiwan \\ ${ }^{2}$ Institute of Biomedical Engineering, National Taiwan University, Taipei, Taiwan \\ E-mail: yychen@cc.ee.ntu.edu.tw
}

Received 6 June 2002, in final form 18 November 2002

Published 23 December 2002

Online at stacks.iop.org/PMB/48/167

\begin{abstract}
This paper investigates the feasibility of conformal heating for external ultrasound hyperthermia by using a phased array transducer with mechanical motion. In this system, a one-dimensional phased array is arranged on a shaft and moves along the shaft, while dynamically focusing on the planning target volume (PTV) with numerous focal spots. To prevent overheating in the intervening tissue between the skin and the PTV, the shaft and the phased array are rotated together to enlarge the acoustical window. With the purpose of conformal heating, the power deposition of the PTV is constructed by combinations of the focal spots and an iterative gradient descent method is then used to determine an optimal set of power weightings for the focal spots. Different tumour shapes are evaluated and the simulation results demonstrate that the volume percentage of the PTV with temperatures higher than $43{ }^{\circ} \mathrm{C}$ is over $95 \%$. The overheating volume outside the PTV is less than $25 \%$ of the PTV. This method provides good conformal heating for external ultrasound hyperthermia. The concept of combining electrical focusing and mechanical motion has the advantages of both enlarging the acoustic window and providing dynamic focusing ability, which is essential for successful conformal heating.
\end{abstract}

\section{Introduction}

Hyperthermia is the process of heating a planning target volume (PTV) to a therapeutic temperature (higher than $42^{\circ} \mathrm{C}$ ) to kill cancerous cells and damage tumour tissues. Numerous investigations (Overgaard 1989, Bornstein et al 1993, Van der Zee et al 2000) indicate that hyperthermia can also act as an auxiliary therapy to radiotherapy, chemotherapy, etc, due to 
its significant enhancement of these therapies. Clinical goals of hyperthermia are to produce a heating volume with a temperature higher than the therapeutic level, conforming to the PTV shape, and to minimize overheating of the non-planning target volume (NPTV) by delivering an appropriate power deposition.

Ultrasound heating is the most attractive method of delivering energy deep within the body due to its penetration and focusing abilities. However, the ultrasound energy is absorbed along the propagation path and may result in overheating of normal tissue in the intervening region between the skin and the PTV. Furthermore, inadequate heating strategies leave cold spots in the PTV while overheating normal tissues, which strongly limits the effectiveness of hyperthermia treatment.

During the past two decades, mechanical, scanned focused ultrasound systems (SFUSs) have been clinically used for treating superficial and deep target volumes (Hand et al 1992, Hynynen et al 1987). A SFUS for deep heating typically includes several spherically focused transducers arranged to form an overlapping focal zone. The focal zone is mechanically scanned in a circular motion to produce a desired heating pattern. Yet, these systems lack the flexibility required to produce heating patterns that conform to various tumour shapes. An improved method scans the focus of the focused transducer throughout the whole tumour. In such a manner, an accurate and desirable heating pattern is obtained. The main feature of this method is precise spatial control of foci distribution, which is based on a computer-controlled, mechanical driving system. To perform this method, multi-degree mechanical motions are employed. However, complex trajectory computations and mechanical motion control are required to avoid undesired power deposition.

Phased array transducers have the potential advantage of steering ultrasound energy without the mechanical motion of transducers. Another significant advantage of phased arrays is that they can be used to directly synthesize heating patterns tailored to various tumour shapes. Several phased array transducers have been developed, including concentric ring arrays (Ibbini and Cain 1990), sector-vortex phased arrays (Umemura and Cain 1989), spherical (Ebbini and Cain 1988) and cylindrical section arrays (Ebbini and Cain 1991), to name a few. However, in spite of the many advantages of phased arrays the dimension of phased array transducers is limited. Lin et al (1999) examines the relationship between the acoustic aperture size and tumour conditions for external ultrasound hyperthermia. Therein, simulation results demonstrate that tumour size, tumour depth and ultrasonic attenuation are the major factors affecting the aperture diameter of the ultrasonic beam required to obtain an appropriate temperature distribution. Clearly, if the size of the transducer aperture is limited, the size of the treatable region is confined. A hybrid technique combining a phased array transducer with mechanical motion may be an appropriate solution for this problem.

In this study, we use a simulation to investigate the feasibility of conformal heating by using a phased array transducer with mechanical motion. Three-dimensional (3D) ultrasound fields and thermal models are developed to simulate the temperature responses produced by this system. For this proposed system, a one-dimensional (1D) phased array forms multiple focal spots on a two-dimensional (2D) plane. As the array shifts along a shaft by mechanical motion, focal spots are deposited in a $3 \mathrm{D}$ volume. Rotating the array and the shaft produces a much larger acoustic window (aperture) to reduce the overheating of normal tissue. An iterative gradient descent method is used to determine the optimal set of power weightings of the focal spots in the desired heating volume. Indices are used to evaluate the heating results for different types of tumours. Simulation results show that appropriate conformal heating is obtained for different tumour and blood perfusion conditions. 


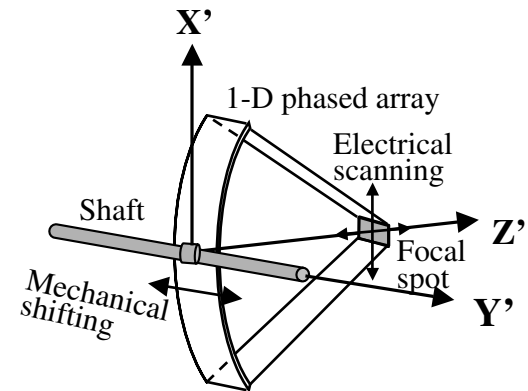

(a)

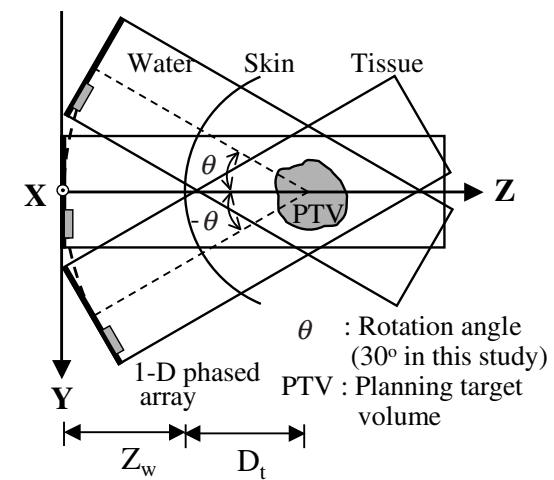

(b)

Figure 1. System configuration of the scanned phased array system. (a) Ultrasound power is emitted from the $1 \mathrm{D}$ phased array transducer and electrically focused on the $X^{\prime}-Z^{\prime}$ plane. The transducer array was shifted along the shaft to produce 3D heating for the entire PTV. (b) The scanning shaft and the transducer array were rotated at $\theta=-30^{\circ}, 0^{\circ}$ and $+30^{\circ}$ back and forth to enlarge the acoustical window. At every rotation angle, the transducer array dynamically focused on the $X^{\prime}-Z^{\prime}$ plane at each position on the shaft. The water-tissue interface was located at $Z_{\mathrm{w}}$ and the centre of the PTV was at a distance $D_{\mathrm{t}}$ away from the interface.

\section{Methods}

\subsection{System description}

A schematic diagram of the proposed ultrasound heating system is shown in figure 1 . The system consists of a 1D phased array transducer and two mechanical motion control mechanisms. Figure 1(a) shows that the phased array is arranged on a shaft and can move along the shaft. By selecting a set of appropriate driving signals, the ultrasound energy is dynamically focused on the $X^{\prime}-Z^{\prime}$ plane. As the phased array is mechanically shifted along the shaft it produces focal spots in a 3D space. To enlarge the acoustical window to deliver ultrasound power, the shaft with the phased array is sequentially rotated at $\theta=-30^{\circ}, 0^{\circ}$ and $+30^{\circ}$, as shown in figure $1(\mathrm{~b})$. Therefore, this heating system is capable of dynamically focusing the ultrasound power on the PTV with numerous focal spots. By selecting a set of appropriate power weightings for these focal spots, a conformal heating pattern is obtained. We assume that the entire system is arranged in a tank of distilled and degassed water, and that the water fills the space between the transducer and the skin. The water-skin interface is located at a distance $Z_{\mathrm{w}}$ away from the transducer's surface and the tumour's centre is at a distance $D_{\mathrm{t}}$ away from the interface.

\subsection{Focal spot distribution}

In a treatment, a number of focused spots are sequentially sonicated to produce the desired heating patterns. The distributions of usable focal spots in this study are shown in figure 2. Figure 2(a) shows the focal spot distribution produced by the phased array at each position on the shaft. The spots are distributed from $-2 \mathrm{~cm}$ to $+2 \mathrm{~cm}$ along the $X^{\prime}$-axis with a $0.5 \mathrm{~cm}$ spacing, and $13-19 \mathrm{~cm}$ along the $Z^{\prime}$-axis with a $1.0 \mathrm{~cm}$ spacing (figure $2(\mathrm{a})$ ). To shorten the complete scanning cycle, the transducer is sonicated by single (layer at $X=0 \mathrm{~cm}$ ) or dual (layers at $X= \pm 0.5, \pm 1.0, \pm 1.5$ and $\pm 2.0 \mathrm{~cm}$ ) focusing modes to produce fundamental focused fields. The phased array is shifted from $-3 \mathrm{~cm}$ to $+3 \mathrm{~cm}$ in the $Y^{\prime}$-direction, with 


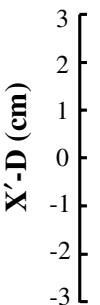

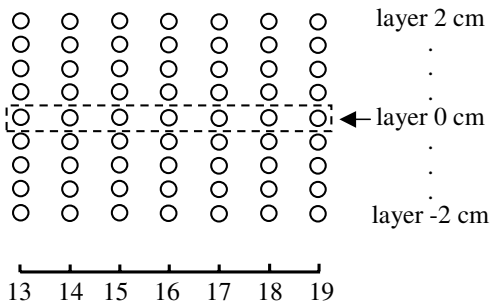

$\mathbf{Z}^{\prime}-\mathbf{D}(\mathbf{c m})$

(a)

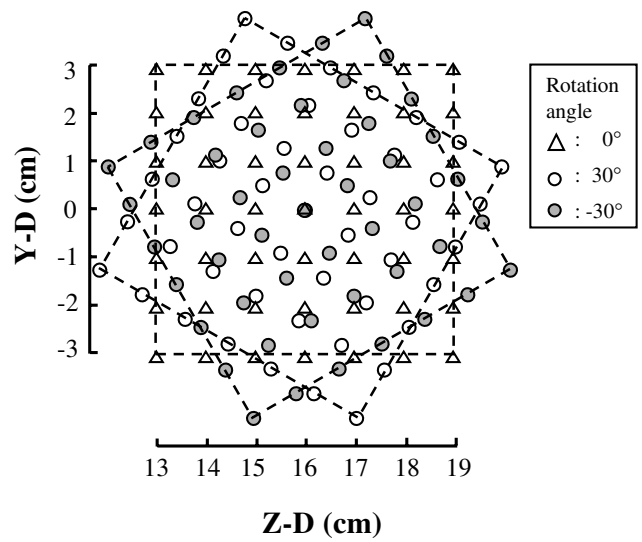

(b)

Figure 2. Placements of focal spots. (a) At a position on the shaft, the transducer array produces focal spots distributed from $X^{\prime}=-2 \mathrm{~cm}$ to $+2 \mathrm{~cm}$ with a $0.5 \mathrm{~cm}$ spacing, and from $Z^{\prime}=13 \mathrm{~cm}$ to $19 \mathrm{~cm}$ with a $1 \mathrm{~cm}$ spacing; (b) the shaft is rotated at angles of $-30^{\circ}, 0^{\circ}$ and $+30^{\circ}$. At each rotation angle, the transducer array was shifted from $-3 \mathrm{~cm}$ to $+3 \mathrm{~cm}$ in the $Y^{\prime}$-direction with $1 \mathrm{~cm}$ spacing and it produced focal spots distributed, as shown in (a), at each position on the shaft. Therefore, multiple focal planes were produced. The focal spots distribution on each focal plane is shown in (b).

Table 1. Parameters used in acoustic and thermal calculations.

\begin{tabular}{ll}
\hline Tissue parameters & Value \\
\hline Speed of sound, $c$ & $1500 \mathrm{~m} \mathrm{~s}^{-1}$ \\
Tissue density, $\rho$ & $1000 \mathrm{~kg} \mathrm{~m}^{-3}$ \\
Ultrasound absorption coefficient, $\alpha$ & $5 \mathrm{~Np} \mathrm{~m}-1 @ 1 \mathrm{MHz}$ \\
Thermal conductivity, $k$ & $0.5 \mathrm{~W} \mathrm{~m}^{-1}{ }^{\circ} \mathrm{C}^{-1}$ \\
Specific heat (blood), $c_{b}$ & $3770 \mathrm{~J} \mathrm{~kg}^{-1}{ }^{\circ} \mathrm{C}^{-1}$ \\
Blood perfusion, $W$ & $2,5,10 \mathrm{~kg} \mathrm{~m}^{-3} \mathrm{~s}^{-1}$ \\
\hline
\end{tabular}

$1.0 \mathrm{~cm}$ spacing. The shaft is rotated at angles of $-30^{\circ}, 0^{\circ}$ and $+30^{\circ}$ (figure $1(\mathrm{~b})$ ). The resulting focal spot distribution could be thought of as a stack of multiple focal layers. The distribution of focal spots on each focal layer is shown in figure 2(b). Not all of these focal spots are used during a treatment; only properly pre-selected focal spots are used. The optimal power weightings of the selected focal spots are obtained by an optimization algorithm.

\subsection{Ultrasound field calculation}

We assume that $N$ fundamental focused fields are selected to produce a desired heating pattern. The absorbed ultrasound power density pattern in tissues produced by the $i$ th focused field can be obtained from

$$
q_{i}=\frac{\alpha p_{i}^{2}}{\rho c}, \quad i=1, \ldots, N
$$

where $\alpha$ is the ultrasound absorption coefficient of tissue, $\rho$ is the tissue density, $c$ is the speed of sound in tissue and $p_{i}$ is the ultrasonic pressure. Typical values for $\alpha, \rho$ and $c$ are used and listed in table 1 . The calculation of ultrasound pressure $p_{i}$ at any field point in 
the tissue is based on Rayleigh-Sommerfeld's diffraction theory. The calculation of driving signals for the transducer elements that produce a specific focused field is conducted by the iterative pseudoinverse method (Ebbini and Cain 1989), which gives driving signals with uniform amplitude and increases the efficiency of the phased array.

\subsection{Thermal model}

The temperature response produced by the $i$ th focused field can be calculated by the steadystate bioheat transfer equation (BHTE) (Pennes 1948)

$$
k \nabla^{2} T_{i}-W c_{b}\left(T_{i}-T_{a}\right)+q_{i}=0
$$

where $k$ is the thermal conductivity of tissue, $T_{a}$ is the arterial temperature $\left(37^{\circ} \mathrm{C}\right), W$ is the blood perfusion and $c_{b}$ is the specific heat capacity of blood. Typical values used are listed in table 1. The values listed are representative of the published data (Duck 1990). The power deposition patterns obtained from equation (1) are substituted into the BHTE and the 3D finite-difference technique is used to find the temperature response. To simplify the calculation, uniform blood perfusion and thermal conductivity are assumed for both normal and tumourous tissues.

\subsection{Scanning}

The ultrasound phased array is periodically shifted and rotated, and the focal zone is sequentially scanned to produce the pre-selected focused fields. If the mechanical motion and scanning speed are fast enough, the spatial superposition of a set of temperature responses from $N$ focused fields approximates that from scanning models (McGough et al 1992). Hence, the temperature response produced by a scanning system is approximated as

or

$$
\boldsymbol{T}_{M \times 1}=\frac{1}{N} \boldsymbol{B}_{M \times N} \boldsymbol{U}_{N \times 1}
$$

$$
\left[\begin{array}{c}
T_{1} \\
T_{2} \\
\vdots \\
T_{M}
\end{array}\right]_{M \times 1}=\frac{1}{N}\left[\begin{array}{cccc}
b_{1,1} & b_{1,2} & \cdots & b_{1, N} \\
b_{2,1} & \ddots & & \vdots \\
\vdots & & b_{j, i} & \vdots \\
b_{M, 1} & \cdots & \cdots & b_{M, N}
\end{array}\right]_{M \times N}\left[\begin{array}{c}
u_{1} \\
u_{2} \\
\vdots \\
u_{N}
\end{array}\right]_{N \times 1}
$$

Here, $\boldsymbol{T}$ describes the approximate temperature response at $M$ discrete field points. $\boldsymbol{B}$ specifies the temperature elevation matrix, where the temperature rise is produced by $N$ focused fields at $M$ discrete field points. Matrix element $b_{j, i}$ represents the temperature generated at the $j$ th field point by the $i$ th focused field. $U$ is the weighting vector of $N$ focused fields.

\subsection{Iterative gradient descent optimization}

The goal of optimization is to find an appropriate weighting vector $\boldsymbol{U}$ to elevate the PTV to the therapeutic temperature $\left(43^{\circ} \mathrm{C}\right.$ in this study) and minimize damage in the normal tissue. McGough et al (1992) proposed an inverse thermal calculation algorithm that determines an appropriate power pattern to approximate the desired temperature field distribution. However, this method yields negative power deposition weightings. If negative power weightings were allowed, this algorithm yields the desired, optimum result. Unfortunately, ultrasound power can never be negative. In this study, a simple, iterative, gradient-based optimization with a penalty function (Gottfried and Weisman 1973) is used to find an optimal solution for this 
non-negative-constrained problem. We define the cost function (Nikita et al 1993, Das et al 1999) as

$$
J=J_{1}+J_{2}+K \cdot \sum_{i=1}^{N} f\left(u_{i}\right)
$$

where

$$
\begin{aligned}
J_{1} & =\sum_{j}\left(T_{\mathrm{s}}-T_{j}\right)^{2}, \quad \text { for } \quad T_{j} \in \mathrm{PTV} \quad \text { and } T_{j}<T_{\mathrm{s}} \\
J_{2} & =\sum_{j}\left(41.5^{\circ} \mathrm{C}-T_{j}\right)^{2}, \quad \text { for } T_{j} \in \mathrm{NPTV} \text { and } T_{j}>41.5^{\circ} \mathrm{C} \\
f\left(u_{i}\right) & = \begin{cases}0, & u_{i} \geqslant 0 \\
u_{i}^{2}, & u_{i}<0\end{cases}
\end{aligned}
$$

where $J_{1}$ is the cost for the temperatures within the PTV lower than the setting temperature $\left(T_{\mathrm{S}}\right)$. The setting temperature can be a parameter used to control the size of the heating region with a temperature higher than the therapeutic level. $J_{2}$ is the cost for the temperatures within the NPTV higher than $41.5^{\circ} \mathrm{C}$. It has been shown that hyperthermia kills cells at temperatures above $42^{\circ} \mathrm{C}$. Therefore, to prevent damage to normal tissue, the maximum allowed temperature for normal tissue is lower than $42{ }^{\circ} \mathrm{C}$. In this study, a more conservative limitation of $41.5^{\circ} \mathrm{C}$ is used. The temperature in the NPTV is expected to be below $41.5^{\circ} \mathrm{C}$. $f\left(u_{i}\right)$ is a penalty term that forces the resulting $i$ th power weighting to be positive. $f\left(u_{i}\right)$ is zero when the $i$ th power weighting is positive. $K$ is a constant that emphasizes the penalty. A suitable value of $K$ should be selected to avoid both the appearance of negative weightings and the dominance of the penalty term in the cost. In our simulation, a value of 1000 is used. The iterative algorithm to find the optimal weighting set is

$$
u_{i}^{n+1}=u_{i}^{n}+\eta \Delta u_{i}^{n}
$$

with

$$
\Delta u_{i}=-\frac{\partial J}{\partial u_{i}}
$$

where $n$ is the iteration number. The $n$th estimated set of weightings is updated to the $(n+1)$ th estimate by the increment $\eta \Delta u_{i} . \eta$ is a constant used to determine the step size of the iteration and a value of $10^{-4}$ is used. About 2000 to 4000 iterations are required for the algorithm to converge for various simulations in this study. At the end of the iterations, a few small negative weightings often remain. These negative weightings can be neglected and set to zero, as no significant effects were observed.

\section{Results}

The ultrasound field model for simulation is a simple water-tissue medium. The centre of the PTV is located at a depth $8 \mathrm{~cm}$ away from the water-skin interface $\left(D_{\mathrm{t}}=8 \mathrm{~cm}\right)$, which is $8 \mathrm{~cm}$ away from the centre of the transducer $\left(Z_{\mathrm{w}}=8 \mathrm{~cm}\right)$, as shown in figure $1(\mathrm{~b})$. The transducer consists of 64 elements, each $3 \mathrm{~mm}$ high and $20 \mathrm{~mm}$ wide, resulting in a $192 \mathrm{~mm}$ arc length (where gaps between elements are ignored). In this study, an $18 \mathrm{~cm}$ radius of curvature of the transducer is used as it produced less undesirable constructive interference. 


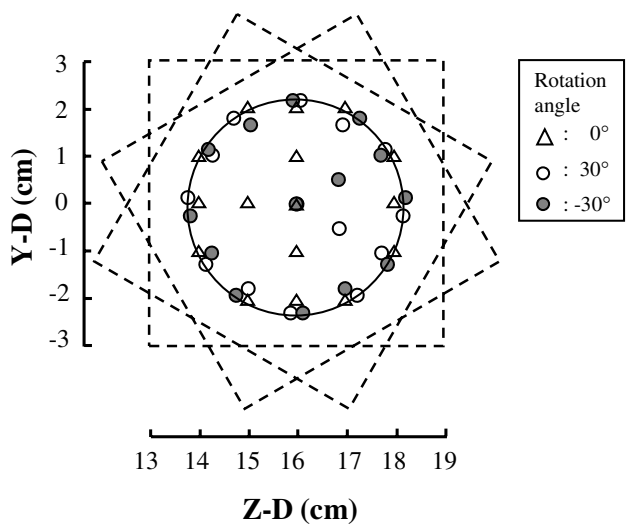

Figure 3. Pre-selected focal spots at each of the focal layers for the simulation of a $2 \mathrm{~cm}$ radius, $4 \mathrm{~cm}$ high cylindrical PTV. In this simulation, focal layers are distributed at $X=0, \pm 1$ and $\pm 2 \mathrm{~cm}$. The solid line represents the boundary of the PTV.

\subsection{Cylindrical heating volume}

In this simulation, the PTV has a radius of $2 \mathrm{~cm}$ and a height of $4 \mathrm{~cm}$, and the blood perfusion for both the PTV and the NPTV is $5 \mathrm{~kg} \mathrm{~m}^{-3} \mathrm{~s}^{-1}$. The first step in simulation is to properly preselect the focal spots. The strategy of selecting focal spots is based on the study of Ocheltree and Frizzell (1987). Their theoretical results indicate that the power deposition required for a tumour treatment can be divided into three regions: the boundary, the outside and the inside of the tumour. Outside the tumour, no power deposition is needed; within the tumour, a constant power equivalent to the power dissipated by blood perfusion is required. At the tumour-normal tissue boundary, a greater power deposition is needed to overcome the strong thermal conduction at the boundary. Therefore, the selected focal spots in this simulation are those distributed near the PTV-NPTV boundary, as well as within the PTV. Figure 3 shows the distribution of selected focal spots at each focal layer. Only five focal layers $(X=0, \pm 1$ and $\pm 2 \mathrm{~cm}$ in figure $2(\mathrm{a}))$ are used.

Figure 4 shows the resulting temperature response for a $T_{\mathrm{s}}$ of $43{ }^{\circ} \mathrm{C}$. Figure 4 (a) shows the $3 \mathrm{D}, 43{ }^{\circ} \mathrm{C}$, isotherm surface, and figures 4 (b) and (c) show the temperature contour on the $X=0, Y=0$ and $Z=16 \mathrm{~cm}$ planes, respectively. It can be seen that the $43{ }^{\circ} \mathrm{C}$ contour (indicated as a shaded area) almost completely covers the PTV region (region demarcated by the dashed line), though a portion of the normal tissue is damaged. Due to the highly focused characteristic of the phased array transducer, the size of focal spots in the $X$-direction is small $(\sim 2 \mathrm{~mm})$. In comparison, the spacing between each focal layer is larger $(\sim 1 \mathrm{~cm})$. Higher ultrasound power for each focal layer is required to elevate the temperature of tissue between each focal layer to $T_{\mathrm{s}}$ by heat conduction. Figure 4(a) shows the correlation with the distribution of pre-selected focal layers. Significant undesired overheating in intervening tissue between the PTV and the skin is not observed.

The converge index (CI) and the external-volume index (EI) (Saw 1988) are commonly used indices in brachytherapy. In this study, these two indices are modified and used for quantitative evaluation of hyperthermia treatments. $\mathrm{CI}\left(T_{\text {index }}\right)$ and $\mathrm{EI}\left(T_{\text {index }}\right)$ can be expressed as

$$
\mathrm{CI}\left(T_{\text {index }}\right)=\frac{\left.\mathrm{PTV}\right|_{\text {Temperature }} \geqslant T_{\text {index }}}{\mathrm{PTV}}
$$



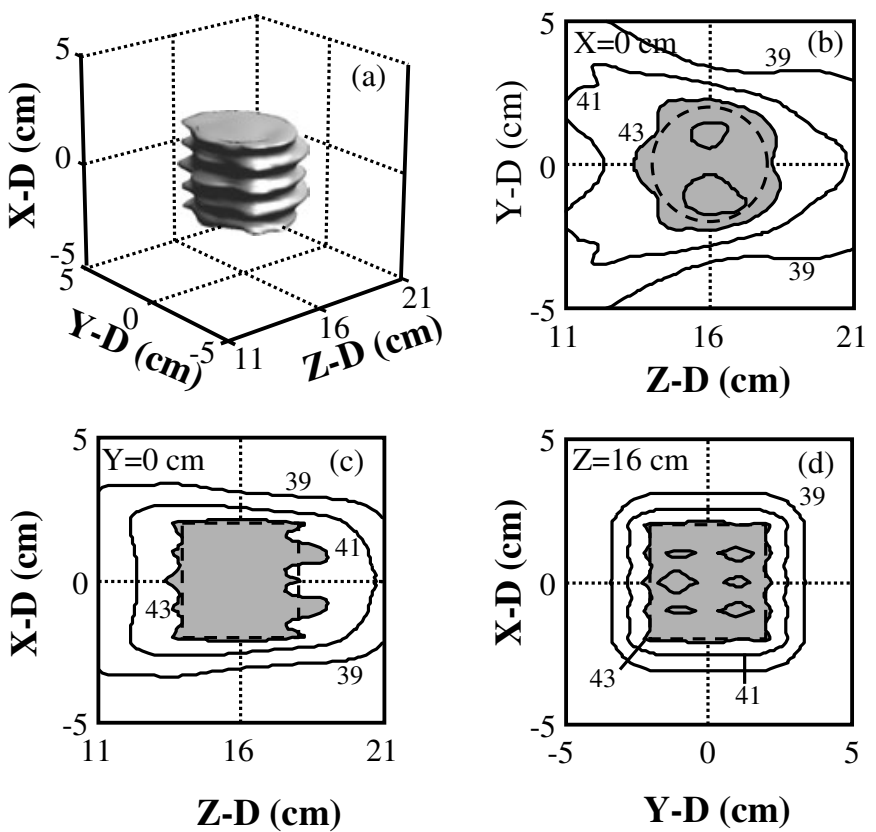

Figure 4. Heating results for the cylindrical PTV with $2 \mathrm{~cm}$ radius and $4 \mathrm{~cm}$ height produced by the scanned phased array system with selected focal spots in figure 3 and a setting temperature $\left(T_{\mathrm{S}}\right)$ of $43{ }^{\circ} \mathrm{C}$ : (a) $3 \mathrm{D} 43{ }^{\circ} \mathrm{C}$ isotherm surface, and temperature contour in (b) $X=0$ plane, (c) $Y=0$ plane and (d) $Z=16 \mathrm{~cm}$ plane. The dashed line represents the boundary of the PTV and the shaded area represents the region with a temperature higher than $43{ }^{\circ} \mathrm{C}$, while each contour within the shaded area increases by $1{ }^{\circ} \mathrm{C}$.

and

$$
\mathrm{EI}\left(T_{\text {index }}\right)=\frac{\left.\mathrm{NPTV}\right|_{\text {Temperature }} \geqslant T_{\text {index }}}{\mathrm{PTV}}
$$

where $\mathrm{CI}\left(T_{\text {index }}\right)$ is defined as the fractional volume of the PTV elevated higher than an index temperature $T_{\text {index }}$ and $\operatorname{EI}\left(T_{\text {index }}\right)$ is defined as the ratio of the volume of the NPTV with temperature higher than $T_{\text {index }}$ to the volume of the PTV.

Figure 5 shows CI and EI curves as a function of $T_{\text {index }}$, which are used for evaluating the simulation results in figure 4 . As shown, $\mathrm{CI}$ and EI decrease with a higher $T_{\text {index }}$. At a temperature of $43{ }^{\circ} \mathrm{C}, 95.3 \%$ of PTV is elevated above $43{ }^{\circ} \mathrm{C}$, while a volume equal to $10.5 \%$ of PTV volume in the NPTV is heated above this temperature. Moreover, the curves indicate the maximum temperatures in the PTV and the NPTV when the CI and EI curves are equal to zero. The maximum temperature inside the PTV is less than $44.5^{\circ} \mathrm{C}$ and the volume with a temperature higher than $44{ }^{\circ} \mathrm{C}$ is less than $5 \%$ of the PTV. This shows a good uniformity of temperature distribution in this treatment.

Variations in blood perfusion within tissues may cause substantial changes to the temperature distribution. Figure 6 shows the temperature distribution in the central axis of the PTV in figure 4 for different blood perfusions. For a small perfusion level $\left(2 \mathrm{~kg} \mathrm{~m}^{-3} \mathrm{~s}^{-1}\right)$, the temperature curve is smoother and more uniform. For a high perfusion level $\left(10 \mathrm{~kg} \mathrm{~m}^{-3} \mathrm{~s}^{-1}\right)$, the temperature curve becomes more unevenly distributed, however the low temperatures of the curve within the PTV are still higher than $43{ }^{\circ} \mathrm{C}$. 


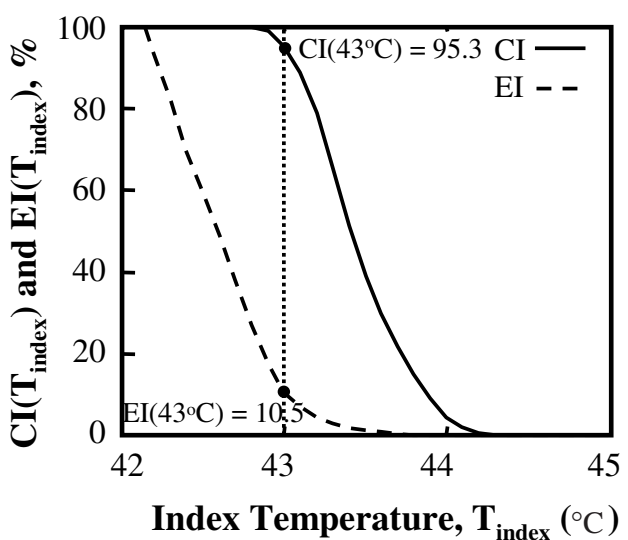

Figure 5. CI and EI as a function of $T_{\text {index }}$ for the simulation in figure 4 .

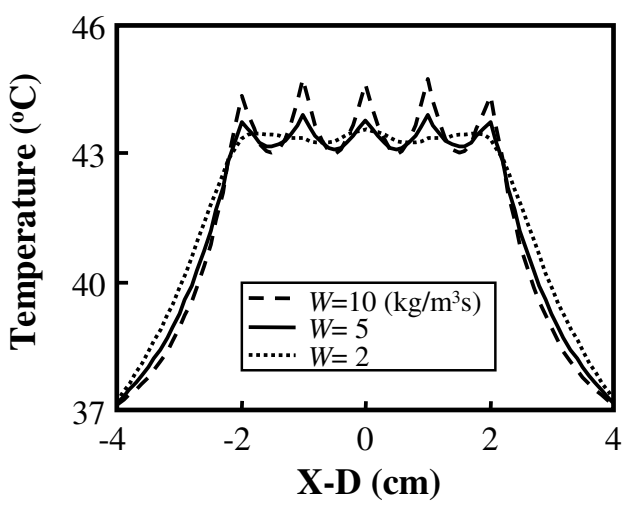

Figure 6. Resulting temperature distribution along the central axis $(Y=0, Z=16 \mathrm{~cm})$ of the cylindrical PTV in figure 4 for blood perfusion 2,5 and $10 \mathrm{~kg} \mathrm{~m}^{-3} \mathrm{~s}^{-1}$.

\subsection{Transient response}

Figure 7 shows the transient temperature responses for the case in figure 4 with various pulse durations, i.e., the sonication time of each focused field for scanning. Each focused field is assumed to have the same pulse duration, and is periodically and sequentially scanned. Figures 7(a)-(c) show temperature distributions on the $X=0$ plane for pulse durations $0.2 \mathrm{~s}$, $0.5 \mathrm{~s}$ and $1.0 \mathrm{~s}$, respectively. As expected, transient temperature responses with smaller pulse durations, implying faster scanning speeds, result in data that are comparable to those produced by the superposition-approximated method. During scanning, focused fields are sequentially excited. Hence, a sharp temperature increase results when the ultrasound power passes a specific location, after which a subsequent temperature decay occurs while other parts of the target volume are scanned. Figure 7(d) shows the maximum and minimum temperatures recorded at the centre of the PTV (point A, $(X, Y, Z)=(0,0,16))$ and at the centre of the top of the PTV (point $\mathrm{B},(X, Y, Z)=(2,0,16))$ versus various pulse durations after the settling state is reached. The magnitude of temperature fluctuation at point $B$ is greater than that at point A due to the stronger thermal conductivity. Furthermore, the magnitude of the temperature fluctuation is linearly dependent on pulse duration. This result agrees with a study by Moros et al (1988) which states that temperature fluctuation is linearly related to scan time. 

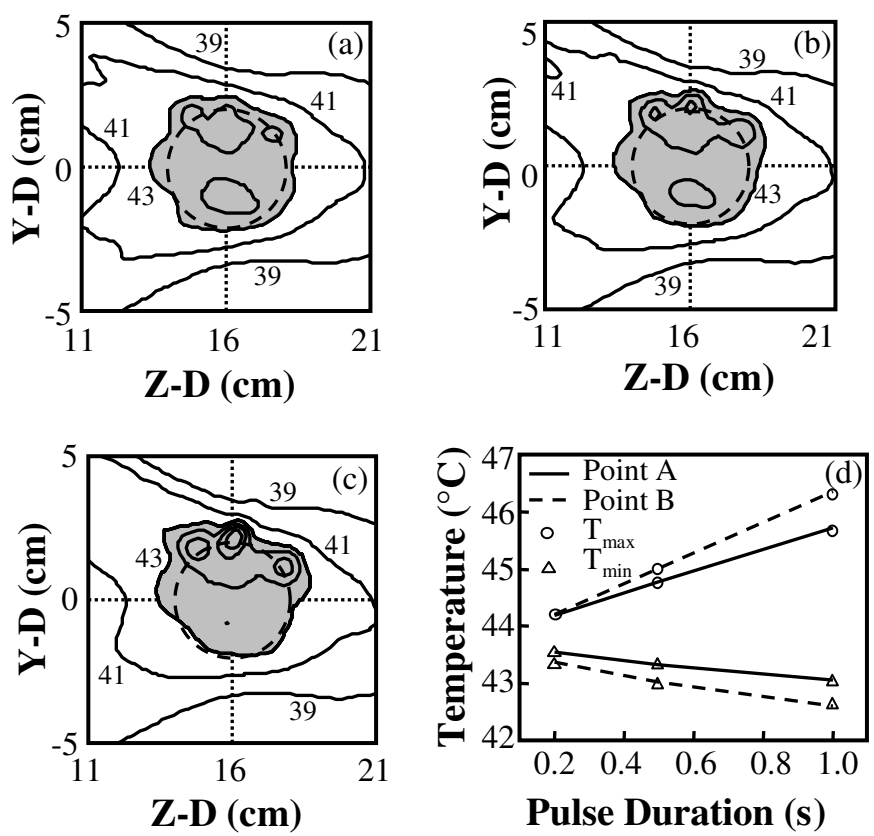

Pulse Duration (s)

Figure 7. Transient responses at the $X=0$ plane for the case in figure 4 with various pulse durations: (a) $0.2 \mathrm{~s}$, (b) $0.5 \mathrm{~s}$ and (c) $1.0 \mathrm{~s}$ for each field pattern in a settling situation. The dashed line represents the boundary of the PTV and the shaded area represents the region with temperature higher than $43{ }^{\circ} \mathrm{C}$, while each contour within the shaded area increases by $1{ }^{\circ} \mathrm{C}$. (d) Shows the maximum $\left(T_{\max }\right)$ and minimum $\left(T_{\min }\right)$ values of the fluctuating temperature at point $A$ (centre of PTV, depicted by a solid line) and point $B$ (centre of the top of PTV, depicted by the dashed line) versus various pulse durations.

\subsection{Effect of setting temperature $\left(T_{\mathrm{s}}\right)$}

In the simulation results in figure $4,95.3 \%$ PTV volume and the volume of $10.5 \%$ of the PTV in the NPTV are elevated above $43{ }^{\circ} \mathrm{C}$. However, in clinical use, physicians may expect to heat the entire PTV above $43{ }^{\circ} \mathrm{C}$ to prevent the presence of under-dosed regions in the tumour tissue. On the other hand, one may expect no tissue in the NPTV to be heated to $43{ }^{\circ} \mathrm{C}$ to avoid the risk of damage to critical organs. The setting temperature $\left(T_{\mathrm{s}}\right.$ in equation (4)) can be used to control the size treatment volume with a temperature higher than $43{ }^{\circ} \mathrm{C}$. Figure 8 shows the values of CI and EI at various $T_{\text {index }}$ as a function of $T_{\mathrm{s}}$. In this simulation, all conditions except $T_{\mathrm{s}}$ are kept the same, as in figure 4 . It is demonstrated that $\mathrm{CI}\left(43{ }^{\circ} \mathrm{C}\right)$ saturated to $100 \%$ when $T_{\mathrm{s}}$ is higher than $43.25^{\circ} \mathrm{C}$ while $\mathrm{EI}\left(43^{\circ} \mathrm{C}\right)$ slowly increased with a higher $T_{\mathrm{s}}$. That is to say, when the temperature of the entire PTV exceeds $43{ }^{\circ} \mathrm{C}$, the resulting cost is to increase the damage volume within the NPTV. $\mathrm{EI}\left(43{ }^{\circ} \mathrm{C}\right)$ decreased to zero when $T_{\mathrm{s}}$ is lower than $42.5^{\circ} \mathrm{C}$, while $\mathrm{CI}\left(43^{\circ} \mathrm{C}\right)$ reduced to about $30 \%$. In this case, no tissue in NPTV is damaged, however the treated volume within the PTV also decreases.

\subsection{Other shapes of heating volume}

For testing the flexibility of this scanned phased array system, figure 9 shows the heating results for different shapes of PTVs for a setting temperature of $43{ }^{\circ} \mathrm{C}$. Figures 9 (a)-(d) display the resulting $3 \mathrm{D}, 43{ }^{\circ} \mathrm{C}$, isotherm surface produced by the scanned phased array system for PTV 


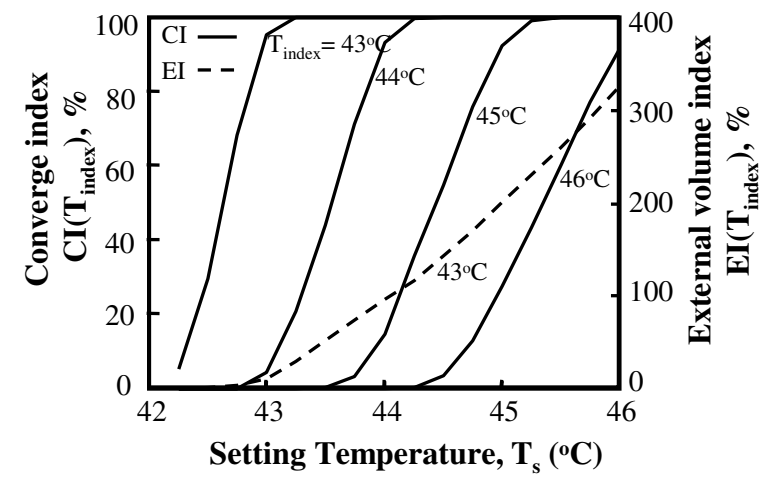

Figure 8. CI and EI for the PTV in figure 4 at different $T_{\text {index }}$ for varying $T_{\mathrm{s}}$. All conditions are kept the same as the simulation in figure 4, except the setting temperature.
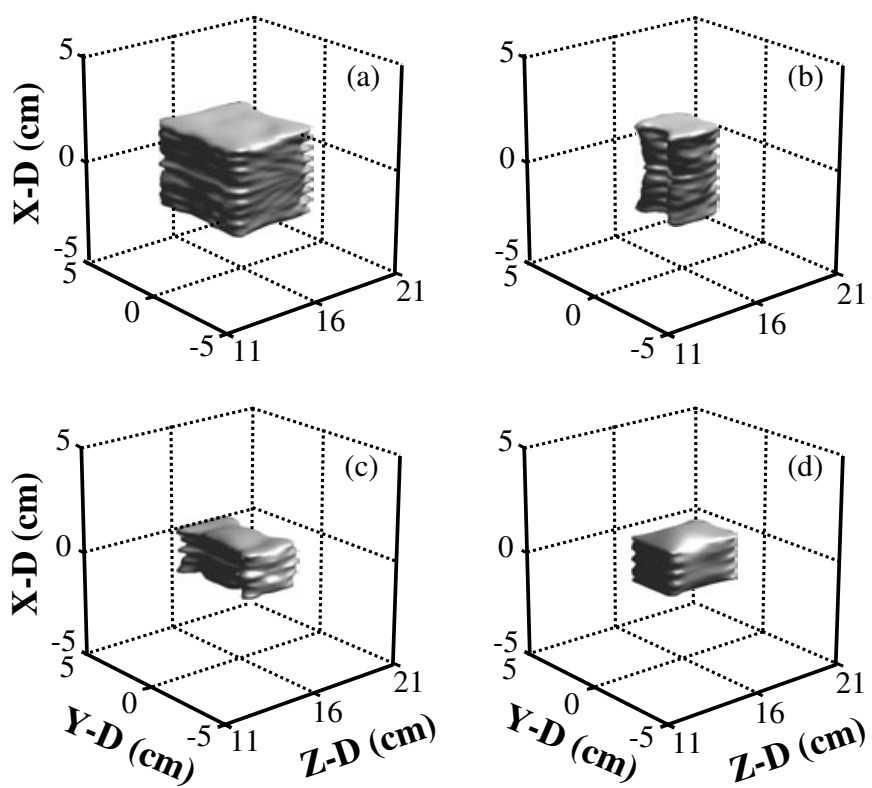

Figure 9. Resulting $43{ }^{\circ} \mathrm{C}$ isotherm plots for (a) $4 \times 4 \times 4 \mathrm{~cm}^{3}$, (b) $4 \times 2 \times 2 \mathrm{~cm}^{3}$, (c) $2 \times 4 \times$ $2 \mathrm{~cm}^{3}$ and (d) $2 \times 2 \times 4 \mathrm{~cm}^{3}$ PTV centred at a depth of $8 \mathrm{~cm}$ under the skin (or $16 \mathrm{~cm}$ from the transducer's surface) produced by the scanned phased array system.

of $4 \times 4 \times 4 \mathrm{~cm}^{3}, 4 \times 2 \times 2 \mathrm{~cm}^{3}, 2 \times 4 \times 2 \mathrm{~cm}^{3}$ and $2 \times 2 \times 4 \mathrm{~cm}^{3}$, respectively. Quantitative indices are listed in table 2 . A rather high $\mathrm{CI}\left(43{ }^{\circ} \mathrm{C}\right)$ value greater than $95 \%$ is obtained with an $\mathrm{EI}\left(43{ }^{\circ} \mathrm{C}\right)$ value less than $25 \%$. Furthermore, in the case of the $2 \times 2 \times 4 \mathrm{~cm}^{3} \mathrm{PTV}$, the maximum temperature is higher than $48^{\circ} \mathrm{C}$, which indicates non-uniformity of temperature distribution within the target volume.

\section{Discussion}

In clinical hyperthermia treatments, we seek to elevate and maintain temperature in the PTV at a therapeutic level, while keeping the temperature rise in the surrounding NPTV as low as 
Table 2. Parameters used and resulting indices for different shapes of target volumes and blood perfusion for the setting temperature of $43{ }^{\circ} \mathrm{C}$.

\begin{tabular}{clllllll}
\hline & $\begin{array}{l}\text { Perfusion } \\
\left(\mathrm{kg} \mathrm{m}^{-3} \mathrm{~s}^{-1}\right)\end{array}$ & $\begin{array}{l}\text { Pulses per } \\
\text { scan cycle }\end{array}$ & Focal layers & $\mathrm{CI}_{43}$ & $\mathrm{EI}_{43}$ & $T_{\max }\left({ }^{\circ} \mathrm{C}\right)$ & $I_{\max }\left(\mathrm{W} \mathrm{cm}^{-2}\right)$ \\
\hline Cylinder & 2 & 81 & $5^{\mathrm{a}}$ & 95.7 & 5.1 & 44 & 153 \\
Radius $=2$ & 5 & 81 & $5^{\mathrm{a}}$ & 95.3 & 10.5 & 44.5 & 269 \\
Height $=4$ & 10 & 83 & $5^{\mathrm{a}}$ & 95.8 & 21.4 & 44.8 & 602 \\
Box $(X \times Y \times Z)$ & & & & & & & \\
$4 \times 4 \times 4$ & 5 & 92 & $9^{\mathrm{b}}$ & 95.4 & 11.6 & 44.7 & 310 \\
$4 \times 2 \times 2$ & 5 & 37 & $9^{\mathrm{b}}$ & 94.3 & 23.0 & 44.0 & 172 \\
$2 \times 4 \times 2$ & 5 & 62 & $5^{\mathrm{c}}$ & 92.4 & 15.8 & 44.4 & 183 \\
$2 \times 2 \times 4$ & 5 & 35 & $5^{\mathrm{c}}$ & 95.1 & 15.7 & 48.3 & 122 \\
\hline
\end{tabular}

${ }^{\mathrm{a}} 0, \pm 1.0, \pm 2.0 \mathrm{~cm}$.

${ }^{\mathrm{b}} 0, \pm 0.5, \pm 1.0, \pm 1.5, \pm 2.0 \mathrm{~cm}$

${ }^{\mathrm{c}} 0, \pm 0.5, \pm 1.0 \mathrm{~cm}$.

$T_{\max }$ : Maximum temperature in tissue.

$I_{\max }$ : Maximum intensity in tissue.

possible. Although a well-designed heating strategy is necessary to accomplish such a goal, a more basic precondition is an acoustic window sufficient for delivering ultrasound power. 2D phased array transducers possess the most flexibility of steering ultrasound power in a 3D space. However, technical limitations constrain the size of the array transducer. Beyond a critical tumour size, pre-focal zone overheating becomes unavoidable (McGough et al 1994). This study combines the advantages of the phased array transducer and mechanical motion to produce a spot scanning power pattern through a fully-utilized acoustic window. Various PTV shapes are tested and simulation results show that the isotherm surface of $43{ }^{\circ} \mathrm{C}$ almost follows the PTV outline. Significant overheating of NPTV regions does not occur in our simulations. This shows the flexibility of the system for producing conformal heating in various PTVs.

In scanned ultrasound hyperthermia systems, temperature at a location periodically raises and abates. To keep the temperature higher than the therapeutic level, greater ultrasound power is needed. Therefore, a significant consideration is the acoustic cavitation in tissue, which leads to mechanical tissue destruction, large temperature rises and reduction of acoustic power transmission. Experimental data (Hynynen 1991) suggest that $700 \mathrm{~W} \mathrm{~cm}^{-2}$ at $1 \mathrm{MHz}$ should be considered as the maximum acceptable ultrasound intensity to avoid unpredictable cavitation damage. In table 2 , we see that in the case of a $4 \times 4 \times 4 \mathrm{~cm}^{3} \mathrm{PTV}$, the maximum intensity for $5 \mathrm{~kg} \mathrm{~m}^{-3} \mathrm{~s}^{-1}$ perfusion simulation is about $310 \mathrm{~W} \mathrm{~cm}^{-2}$. For the simulations of cylindrical PTV with various perfusions, it can be seen that a greater maximum intensity is needed to achieve the same temperature rise for a higher perfusion case. When a high blood perfusion of $10 \mathrm{~kg} \mathrm{~m}^{-3} \mathrm{~s}^{-1}$ is assumed, the maximum intensity in tissue $\left(602 \mathrm{~W} \mathrm{~cm}^{-2}\right)$ approaches the threshold value of cavitation.

Another consideration of a scanning system is fluctuations in temperature elevation. Moros et al (1988) reported that to minimize large transient temperature fluctuations, complete scanning cycles must be less than $\sim 10$ s for scanned, focused, ultrasound hyperthermia systems. This suggested value is significantly dependent on the focal pattern, scanning trajectory and blood perfusion. For the simulation in figure 7(a), 81 focused fields and $0.2 \mathrm{~s}$ pulse duration are used for scanning; and a complete scanning cycle results in $16.2 \mathrm{~s}$. However, the magnitude of temperature fluctuations produced at the centre of the PTV is less than $1{ }^{\circ} \mathrm{C}$ (figure $7(\mathrm{~d})$ ). Even at the centre of the top of the PTV, which exhibits a larger thermal conductive effect, the magnitude is about $1{ }^{\circ} \mathrm{C}$. This value is smaller than that in Moros' study 
$\left(\sim 1.5^{\circ} \mathrm{C}\right)$. This difference is caused by the scanning trajectory and the focusing properties of the transducers used. In their study, a highly focused, spherical transducer is used. The focus is scanned circularly on a single focal plane and less ultrasound energy is overlapped. In our study, the foci are distributed in a 3D volume, and the sizes of the focal zones are large. This results in the ultrasound energy overlap among sonications and smaller temperature fluctuations.

A parameter significant to the magnitude of maximum intensity and temperature fluctuation described above is the number of focused fields selected for scanning. The more focused fields used, the longer the complete scanning cycle becomes. To keep the temperature within the target volume higher than the therapeutic level, spatial and temporal peaks of ultrasound intensity increase, and larger temperature fluctuations follow. For this reason, although there are over a thousand usable focal spots distributed throughout a treatment region, only properly pre-selected spots are used and substituted into the iterative optimization algorithm to find a weighting set for conformal heating. In figure 4, for the cylindrical PTV, to reduce the number of used focused fields, only five focal layers (each $1 \mathrm{~cm}$ apart) are used. However, an uneven heating pattern (figure 4(a)) is obtained. In comparison, a total of nine focal layers (each $0.5 \mathrm{~cm}$ apart) are used for the $4 \times 4 \times 4 \mathrm{~cm}^{3}$ cubic PTV (figure 9(a)) and a smoother temperature distribution is obtained. Thus, there is an evident trade-off problem between uniform heating patterns and small temperature fluctuations.

Several studies (Dewey et al 1977, Fajardo 1984, Field et al 1977) indicate that the effectiveness of hyperthermia is markedly dependent on temperature uniformity. A small variance in temperature can cause a significant difference in cell (normal/tumour) tissue damage. Hence, in hyperthermia, a more uniformly distributed heating pattern within the PTV is desired. A decisive parameter for uniform heating is blood perfusion (figure 6). To accumulate a sufficient thermal dose, the temperature in the PTV should be elevated and maintained at therapeutic levels for a certain period of time. However, over the duration, a homeostatic control mechanism tends to increase the blood flow to remove heat from the heated tissue, which increases the difficulty of hyperthermic treatment. Although some animal trials indicate that blood flow in tumours declines with increasing temperature, no apparent evidence of a sharp reduction in flow is observed in human tumours (Song et al 1995). Blood perfusion of human tissue varies over a wide range of different tissue types. A constant, high perfusion rate of $5 \mathrm{~kg} \mathrm{~m}^{-3} \mathrm{~s}^{-1}$ is assumed in our simulation; two extreme levels (2 and $10 \mathrm{~kg} \mathrm{~m}^{-3} \mathrm{~s}^{-1}$ ) are also tested in some cases (figure 5). In most of our simulations, as shown in table 2, the values of $\mathrm{CI}\left(43{ }^{\circ} \mathrm{C}\right)$ are higher than $95 \%$, even in the high perfusion case. At high perfusion levels, the value of $\mathrm{EI}\left(43{ }^{\circ} \mathrm{C}\right)$ and maximum acoustic intensity in tissue, $I_{\max }$, are large, and an uneven temperature distribution is obtained. One of the reasons is that the larger power is needed to compensate for the heat removed by higher blood perfusion.

In this study, a uniform blood perfusion is assumed for both normal and tumour tissues. However, the perfusion is generally non-uniformly distributed. The study of Endrich et al (1979) indicates that perfusion in a tumour varies from a very small value in the necrotic core region to a relatively high value in the peripheral region. This perfusion difference may result in an unexpected temperature-rise distribution when a uniform perfusion is assumed during the hyperthermia treatment. Hence, a non-uniform perfusion case is also investigated and the result is shown in figure 10. The PTV is a cylinder (with both diameter and height of $4 \mathrm{~cm}$ ) divided into three layers. Blood perfusion from the peripheral layer to the core is 4,2 and $0.5 \mathrm{~kg} \mathrm{~m}^{-3} \mathrm{~s}^{-1}$, respectively. In the surrounding normal tissue, perfusion is assumed to be $2 \mathrm{~kg} \mathrm{~m}^{-3} \mathrm{~s}^{-1}$. Pre-selected focal spots for heating on each focal layer are shown in figure 3, and nine focal layers are used $(X=0, \pm 0.5, \pm 1, \pm 1.5$ and $\pm 2 \mathrm{~cm}$ in figure 2(a)). The heating results show that a greater region in the NPTV is damaged when compared with the uniform 

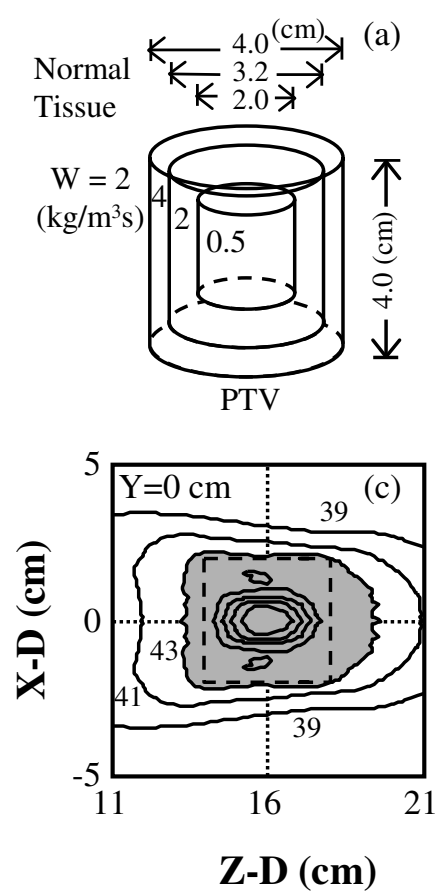
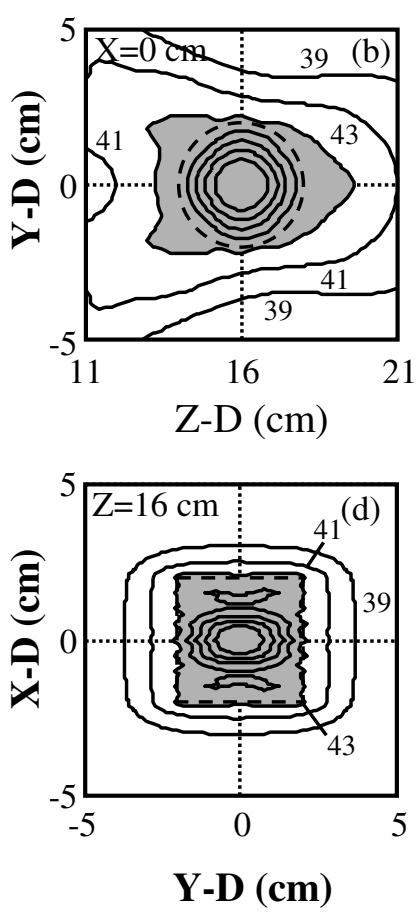

Figure 10. Simulation results for a non-uniform-perfused cylindrical PTV. (a) The cylindrical PTV has both diameter and height of $4 \mathrm{~cm}$ each, and is divided into three layers. Perfusion from periphery to core is 4,2 and $0.5 \mathrm{~kg} \mathrm{~m}^{-3} \mathrm{~s}^{-1}$, respectively. Perfusion in the normal tissue is assumed to be $2 \mathrm{~kg} \mathrm{~m}^{-3} \mathrm{~s}^{-1}$. Temperature distributions are shown in (b) the $X=0$ plane, (c) the $Y=0$ plane and (d) the $Z=16 \mathrm{~cm}$ plane. The dashed line represents the boundary of the PTV and the shaded area is the region with a temperature higher than $43{ }^{\circ} \mathrm{C}$, while each contour within the shaded area increases by $1{ }^{\circ} \mathrm{C}$.

perfusion case. To elevate the temperature in the peripheral region of the PTV, greater power is required to compensate for the convective effect of the higher perfusion condition. This results in more energy accumulation and higher temperature rise in the NPTV than exhibited in the uniform-perfused model. To reduce this problem, an extended acoustical window can be used to average and reduce the accumulated and overlapped power in the NPTV region.

Heating results can be highly dependent on the shape of the PTV. It can be seen from table 2, by using the optimization algorithm, that $\mathrm{CI}\left(43{ }^{\circ} \mathrm{C}\right)$ approaches $95 \%$. However, $\mathrm{EI}\left(43^{\circ} \mathrm{C}\right)$ varies greatly with different PTV shapes. For example, the simulation of the $4 \times 2 \times$ $2 \mathrm{~cm}^{3}$ PTV presents a EI $\left(43{ }^{\circ} \mathrm{C}\right)$ value of $23 \%$, owing to a small effective acoustic window used and more ultrasound energy accumulated in the NPTV. Furthermore, for the $2 \times 2 \times$ $4 \mathrm{~cm}^{3}$ PTV, the maximum temperature is $48.3^{\circ} \mathrm{C}$. The reason can be the longitudinal PTV and, hence, considerable ultrasound energy is accumulated.

\section{Conclusion}

In this study, we investigated the feasibility of an ultrasound hyperthermia system to produce conformal heating. Different shapes of tumours were tested and simulation results show that, in most cases, the percentage of the PTV for successful heating is over $95 \%$ and the damage volume in NPTV is less than $25 \%$ PTV volume. Compared to existing studies, this method 
provides an acceptable conformal heating in ultrasound hyperthermia. The combination of electrical focusing and mechanical motion has the advantages of enlarging the acoustic window as well as providing dynamic focusing ability, which forms the essence of successful conformability.

\section{Acknowledgment}

The authors would like to thank the National Science Council of Taiwan for partially supporting this research under contract no NSC 90-2213-E-002-061.

\section{References}

Bornstein B A, Zouranjian P S, Hansen J L, Fraser S M, Gelwan L A, Teicher B A and Svensson G K 1993 Local hyperthermia, radiation therapy and chemotherapy in patients with local-regional recurrence of breast carcinoma Int. J. Radiat. Oncol. Biol. Phys. 25 79-85

Das S K, Clegg S T and Samulski T V 1999 Computational techniques for fast hyperthermia temperature optimization Med. Phys. 26 319-28

Dewey W C, Hopwood L E, Sapareto S A and Gerweck L F 1977 Cellular responses to combination of hyperthermia and radiation Radiology 123 463-74

Diederich C J and Hynynen K 1999 Ultrasound technology for hyperthermia Ultrasound Med. Biol. 25 871-86

Duck F A 1990 Physical Properties of Tissue (New York: Academic)

Ebbini E S and Cain C A 1988 A cylindrical-section ultrasound phased-array applicator for hyperthermia cancer therapy IEEE Trans. Ultrason. Ferroelectr. Freq. Control 35 561-72

Ebbini E S and Cain C A 1989 Multi-focus ultrasound phased-array pattern synthesis: optimal driving-signal distributions for hyperthermia IEEE Trans. Ultrason. Ferroelectr. Freq. Control 36 540-8

Ebbini E S and Cain C A 1991 A spherical-section ultrasound phased array applicator for deep localized hyperthermia IEEE Trans. Biomed. Eng. 38 634-43

Endrich B, Reinhold H S, Gross J F and Intaglietta M 1979 Tissue perfusion inhomogeneity during early tumor growth in rats $J$. Natl Cancer Inst. 62 387-93

Fajardo L F 1984 Pathological effects of hyperthermia in normal tissue Cancer Res. (Suppl.) 44 4826s-35s

Field S B, Hume S P, Law M P and Myers R 1977 The response of tissues to combined hyperthermia and X-rays $B r$. J. Radiol. 50 129-34

Gottfried B S and Weisman J 1973 Introduction to Optimization Theory (Englewood Cliffs, NJ: Prentice-Hall)

Hand J W, Vernon C C and Prior M V 1992 Early experience of a commercial scanned focused ultrasound hyperthermia system Int. J. Hyperth. 8 587-607

Hynynen K 1991 The threshold for thermally significant cavitation in dog's thigh muscle in vivo Ultrasound Med. Biol. 17 157-69

Hynynen K, Roemer R, Anhalt D, Johnson C, Xu Z X, Swindell W and Cetas T 1987 A scanned, focused, multiple transducer ultrasonic system for localized hyperthermia treatments Int. J. Hyperth. 3 21-35

Ibbini M S and Cain C A 1990 The concentric-ring array for ultrasound hyperthermia: combined mechanical and electrical scanning Int. J. Hyperth. 6 401-19

Lin W L, Yen J Y, Chen Y Y, Jin K W and Shieh M J 1999 Relationship between acoustic aperture size and tumor condition for external ultrasound hyperthermia Med. Phys. 26 818-24

McGough R J, Ebbini E S and Cain C A 1992 Direct computation of ultrasound phased-array driving signals from a specified temperature distribution for hyperthermia IEEE Trans. Biomed. Eng. 39 825-35

McGough R J, Wang H, Ebbini E S and Cain C A 1994 Mode scanning: heating pattern synthesis with ultrasound phased arrays Int. J. Hyperth. $10433-42$

Moros E G, Roemer R B and Hynynen K 1988 Simulation of scanned focused ultrasound hyperthermia: the effects of scanning speed and pattern on the temperature fluctuations at the focal depth IEEE Trans. Ultrason. Ferroelectr. Freq. Control 35 552-9

Nikita K S, Maratos N G and Uzunoglu N K 1993 Optimal steady-state temperature distribution for a phased array hyperthermia system IEEE Trans. Biomed. Eng. 40 1299-306

Ocheltree K B and Frizzell L A 1987 Determination of power deposition patterns for localized hyperthermia: a steady-state analysis Int. J. Hyperth. $3269-76$

Ocheltree K B and Frizzell L A 1988 Determination of power deposition patterns for localized hyperthermia: a transient analysis Int. J. Hyperth. 4 281-96 
Overgaard J 1989 The current and potential role of hyperthermia in radiotherapy Int. J. Radiat. Oncol. Biol. Phys. 16 537-49

Pennes H H 1948 Analysis of tissue and arterial blood temperatures in the resting human forearm J. Appl. Physiol. 1 19-122

Saw C B 1988 Reference dose rates for single- and double-plane ${ }^{192}$ Ir implants Med. Phys. 15 391-5

Song C W, Choi I B, Han B S, Sahu S K and Osborn J L 1995 Microvasculature and perfusion in normal tissue and tumors Thermalradiotherapy and Thermochemotherapy vol 1 ed M H Seegenschmiedt, P Fessenden and C C Vernon (New York: Springer) pp 139-56

Umemura S and Cain C A 1989 The sector-vortex phased array: acoustic field synthesis for hyperthermia IEEE Trans. Ultrason. Ferroelectr. Freq. Control 36 249-57

Van der Zee J, González D, van Rhoon G C, van Dijk J D P, van Putten W L J and Hart A A M 2000 Comparison of radiotherapy alone with radiotherapy plus hyperthermia in locally advanced pelvic tumours: a prospective, randomised, multicentre trial Lancet 355 1119-25 